\title{
Parte IV. Terapia antimicrobiana en pacientes con cáncer y receptores de trasplante de precursores hematopoyéticos
}

\author{
María Elena Santolaya', Ana María Álvarez², Teresa Bidart, Jorge Morales ${ }^{4}$ y Claudio González ${ }^{5}$
}

\section{Antimicrobial therapy in cancer patients and hematopoietic stem cell transplantation receptors}

This manuscript includes the antiinfective therapeutic resources for immunocompromised patients under chemotherapy by cancer or hematopoietic stem cells transplant (HSCT) receptors. The document presents the antimicrobial therapy indicated in the most prevalent clinical situations in this population and the primary and alternative therapy for some specific microorganisms. The clinical situations included in the analysis are: febrile neutropenia without focus, sepsis, infections of the central nervous system, pneumonia, skin and soft tissue infections, neutropenic enterocolitis and urinary tract infection. The therapeutic resources, recommended doses and special precautions for the use of antimicrobial recommended in bacterial, viral, fungal and parasitic infections in this population are described, including the measurement of plasma concentrations of certain drugs in specific situations.

Keywords: Antimicrobial therapy; cancer; neutropenia; febrile neutropenia; hematopoietic stem cell transplantation.

Palabras clave: Terapia antimicrobiana; cáncer; neutropenia; neutropenia febril; trasplante de precursores hematopoyéticos.

\begin{tabular}{|ll|}
\hline Abreviaturas \\
CVC & : Catéter venoso central \\
EFI & : Enfermedad fúngica invasora \\
IBI & : Infección bacteriana invasora \\
IFI & : Infección fúngica invasora \\
IV & : Intravenoso \\
LBA & : Lavado broncoalveolar \\
NF & : Neutropenia febril \\
PCR & : Proteína C reactiva \\
PHO & : Pacientes hemato-oncológicos \\
RAN & : Recuento absoluto de neutrófilos \\
RM & : Resonancia magnética \\
RPC & : Reacción de polimerasa en cadena \\
SNC & : Sistema nervioso central \\
TAC & : Tomografía axial computada \\
TPH & $:$ Trasplante de precursores hematopoyéticos \\
UPC & : Unidad de Paciente Crítico \\
\hline
\end{tabular}

\section{Conceptos generales}

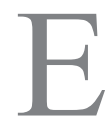

1 manejo actual de los pacientes con neutropenia febril (NF) en pacientes con cáncer o trasplante de precursores hematopoyéticos (TPH), que en su conjunto identificaremos como pacientes hemato- oncológicos (PHO), implica la categorización en alto o bajo riesgo de desarrollar infecciones bacterianas invasoras (IBI), lo que permite implementar estrategias de manejo selectivo ${ }^{1-3}$ (Figura 1).

Todos los pacientes con NF (recuento absoluto de neutrófilos $\leq 500$ céls $/ \mathrm{mm}^{3}$ ) más una medición de temperatura axilar $\geq 38,5^{\circ} \mathrm{C}$ o dos mediciones $\geq 38,0{ }^{\circ} \mathrm{C}$ en un período $>$ a $1 \mathrm{~h}$, deben ser hospitalizados por al menos $24 \mathrm{~h}$, período en que se realiza una evaluación clínica y de laboratorio tendiente a su clasificación de riesgo y a la búsqueda de posibles focos de infección y se inicia terapia antimicrobiana intravenosa (IV) de acuerdo al riesgo de IBI, la epidemiología local de las infecciones y los patrones de susceptibilidad antimicrobiana conocidos de la institución ${ }^{4,5}$.

\section{Evaluación clínica y de laboratorio de ingreso}

La evaluación clínica se orienta a buscar los principales focos causantes de NF: respiratorio alto y bajo, piel y tejidos blandos, digestivo, relacionado al catéter venoso central (CVC), urinario y bacteriemias sin localización.

El examen físico debe evaluar el estado general del paciente, función respiratoria, nivel de consciencia, estabilidad hemodinámica (presión arterial, velocidad de relleno capilar) y signos de gravedad que acompañen a la fiebre. La neutropenia condiciona una respuesta inflamatoria escasa, con una semiología atípica, por lo que el examen
'Unidad de Infectología, Hospital Dr. Luis Calvo Mackenna, Facultad de Medicina, Universidad de Chile. Santiago, Chile. 2Departamento de Pediatría, Hospital San Juan de Dios. Santiago, Chile.

3Departamento de Medicina, Clínica Santa María. Santiago, Chile.

${ }^{4}$ Servicio de Farmacia Clínica, Hospital Dr. Luis Calvo Mackenna. Santiago, Chile.

${ }^{5}$ Servicio de Farmacia Clínica, Hospital Exequiel González Cortés. Santiago, Chile.

Los autores declaran ausencia de conflicto de interés. El presente trabajo no recibió financiamiento alguno.

Correspondencia a: María Elena Santolaya de Pablo msantola@med.uchile.cl 


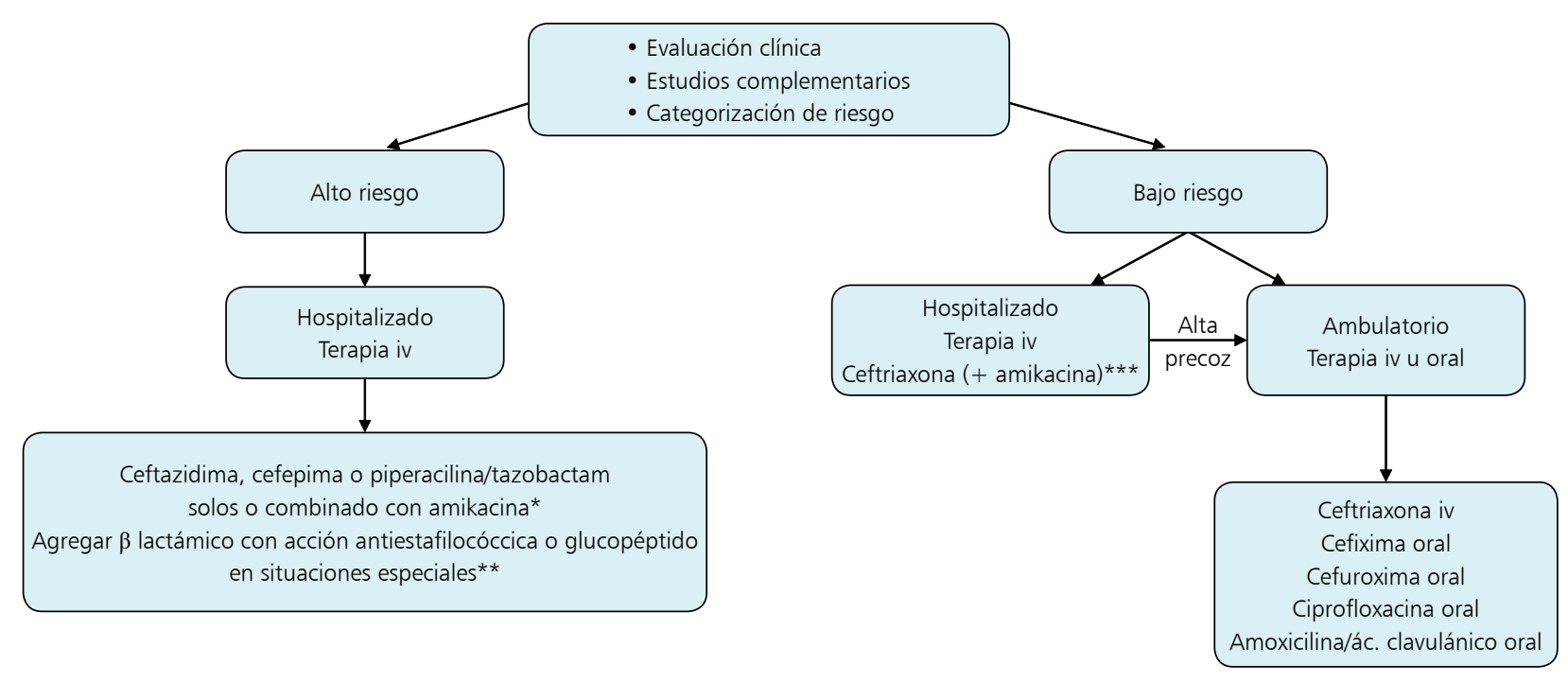

Figura 1. Algoritmo de manejo general del paciente con neutropenia febril. *Uso de aminoglucósidos de acuerdo a epidemiología. **Se recomienda agregar vancomicina a los pacientes con: sospecha clínica de infección asociada a CVC, infección de piel y tejidos blandos en áreas donde exista una tasa de S. aureus resistente a meticilina > al 15\%, infección osteo articular, evidencia de sepsis y bacteriemia por cocáceas grampositivas, previo a la identificación final y evaluación de susceptibilidad. ***Optativo, de acuerdo a la epidemiología.

clínico debe ser exhaustivo y dirigido, especialmente en la semiología de la cavidad oral, la mucosa genital, zonas de inserción y trayecto de catéteres, punciones venosas y el examen pulmonar.

En cuanto a la evaluación de laboratorio, se sugieren los siguientes exámenes mínimos: hemograma, recuento absoluto de plaquetas, análisis de las funciones renal y hepática, PCR cuantitativa y hemocultivos periféricos, y de cada rama, en pacientes con catéter venoso central (CVC) permanente; si es posible de realizar, se pueden agregar examen de orina completa y urocultivo, pero en ningún caso deben retrasar el inicio de la terapia antimicrobiana. Dependiendo de la orientación clínica $\mathrm{y}$ antecedentes epidemiológicos locales, considere la toma de otras muestras para estudios pertinentes: tracto respiratorio, digestivo, sistema nervioso central (SNC), senos paranasales, piel y mucosas, entre otros.

\section{Clasificación de riesgo}

Se han propuesto diferentes modelos de predicción de riesgo en la literatura científica para identificar pacientes con mayor o menor riesgo de desarrollar complicaciones, a fin de plantear su manejo hospitalario o ambulatorio según el respectivo riesgo estimado. En adultos, se sugiere seguir el modelo de MASCC, Talcott o CISNE en conjunto con el criterio clínico ${ }^{6}$, pero en general los pacientes que reciben inducción de leucemia mieloide aguda o TPH no podrán manejarse de manera ambulatoria, aunque los modelos los consideren de bajo riesgo. $\mathrm{MASCC}^{7}$ contempla los siguientes parámetros: edad, estado general, estado de hidratación, presión arterial, presencia de enfermedad pulmonar obstructiva crónica y antecedente de infección fúngica invasora. En niños, se recomienda seguir el modelo desarrollado por el grupo chileno PINDA ${ }^{8}$, que considera las siguientes variables: tipo de cáncer, presión arterial, intervalo entre el término del último ciclo de quimioterapia y el inicio de la fiebre, cuantificación de proteína $\mathrm{C}$ reactiva $(\mathrm{PCR})$ y recuento absoluto de plaquetas.

\section{Neutropenia febril sin foco}

En los pacientes con episodios de bajo riesgo, se recomienda continuar el manejo en forma ambulatoria luego de una hospitalización abreviada ${ }^{6-9}$. En Pediatría se ha validado la suspensión de la terapia antimicrobiana cuando se documenta una infección por un virus respiratorio como agente único en pacientes con evolución clínica favorable ${ }^{10}$.

En los pacientes con NF de alto riesgo, se recomienda continuar el manejo en forma intrahospitalaria hasta su recuperación ${ }^{11,12}$. Es importante identificar, dentro del grupo de pacientes de alto riesgo, aquellos en riesgo de 
sepsis $( \pm 20 \%)$ y los que están en riesgo de fallecer por la gravedad de la infección $( \pm 5 \%)$, para implementar en ellos medidas de manejo más enérgicas, incluido el traslado precoz a Unidades de Paciente Crítico (UPC) ${ }^{13-15}$.

Los criterios de selección y ajuste de antimicrobianos para cada grupo se basan en la presencia o ausencia de foco clínico y en patrones de epidemiología y susceptibilidad bacteriana in vitro de cada institución.

La evaluación de ambos grupos se realiza diariamente. Una vez cumplidas las primeras $72 \mathrm{~h}$ de terapia antimicrobiana, la evolución se cataloga como favorable o desfavorable, de acuerdo a criterios clínicos y de laboratorio establecidos ${ }^{1,8}$. Se considera que un paciente tiene evolución favorable si presenta: estabilización hemodinámica; caída paulatina y significativa de la fiebre; resolución progresiva de focos infecciosos presentes al ingreso y ausencia de nuevos focos de infección; descenso significativo de la PCR.

La mayoría de los pacientes con episodios de NF se encuentran en vías de recuperación de su neutropenia (definida como $\mathrm{RAN}<500 / \mathrm{mm}^{3}$ ) y con curva febril en disminución al cuarto día de evolución. La prolongación de la fiebre y la neutropenia más allá de cuatro días, favorecen el surgimiento de infecciones fúngicas invasoras (IFI), difíciles de objetivar, las que deben ser investigadas exhaustivamente en esta condición ${ }^{16,17}$.

\section{Neutropenia febril y sepsis}

En el contexto del paciente con NF de alto riesgo, alrededor de $20 \%$ puede desarrollar sepsis ${ }^{18}$. El médico clínico que atiende estos pacientes debe estar alerta y buscar exhaustivamente los síntomas y signos sugerentes de sepsis para actuar oportunamente, optimizando la condición hemodinámica del paciente y aporte de oxigenoterapia, asegurando buenos accesos venosos, realizando las maniobras de resucitación y asegurando un acceso oportuno a UPC cuando sea necesario.

Un tema de manejo relevante es iniciar una terapia antimicrobiana empírica, de amplio espectro, antes de una hora de la consulta del paciente al hospital, previa obtención de hemocultivos (periféricos y centrales si existe (VC) ${ }^{19,20}$.

La terapia antimicrobiana empírica se indica de acuerdo a la historia del paciente con cobertura para bacilos gramnegativos incluyendo Pseudomonas sp, más cobertura para cocáceas grampositivas si existe sospecha de infección del CVC, piel, tejidos blandos, neumonía o inestabilidad hemodinámica. Si hay evidencia de mucositis necrosante, sinusitis, celulitis odontógena, celulitis perirrectal o infección intraabdominal, incluyendo enterocolitis, se sugiere adicionar cobertura para bacterias anaerobias estrictas (Tabla 1$)^{21-23}$.

\section{Infecciones del sistema nervioso central}

Los factores de riesgo para presentar infecciones del sistema nervioso central (SNC) son fundamentalmente la neutropenia profunda y prolongada y la terapia inmunosupresora intensiva. ${ }^{24}$

Los PHO son susceptibles a contraer infecciones del SNC por diferentes microorganismos: virus, bacterias, hongos y parásitos. Las estrategias de quimioprofilaxis en el paciente con TPH han provocado un cambio en la epidemiología de las infecciones oportunistas del $\mathrm{SNC}^{25}$.

Las manifestaciones clínicas no son específicas, pueden incluir fiebre, cefalea, alteración del estado mental, signos focales neurológicos, convulsiones, etc. Las imágenes cerebrales ayudan a clasificar las infecciones del SNC en dos entidades principales: lesiones focales (absceso cerebral o lesiones nodulares) y meningitis o meningo-encefalitis.

Dentro de las lesiones focales, las etiologías más frecuentes son Aspergillus spp y Toxoplasma gondii, seguido de zygomicosis, infecciones por Nocardia spp, virus de Epstein Barr y amebas de vida libre ${ }^{26}$.

Dentro de las meningitis o meningoencefalitis, las etiologías más frecuentes son Cryptococcus neoformans, Listeria monocytogenes $y$ virus herpes simplex, seguido de citomegalovirus (CMV), virus varicela zoster (VVZ), virus herpes 6 , poliomavirus (JC, BK) y amebas de vida libre; la frecuencia de infección del SNC por Mycobacterium spp dependerá de la epidemiología existente en el entorno del paciente $y / o$ lugar de su procedencia, pudiendo adoptar la forma focalizada o diseminada ${ }^{27,28}$.

La infección del SNC en PHO es una emergencia médica. La evaluación debe ser guiada por el grado de compromiso del sistema inmune. Si el paciente es un receptor de TPH, considere el tipo de trasplante, el tiempo transcurrido desde el procedimiento, el estado serológico del donante y receptor, adherencia a profilaxis y los síntomas sistémicos concomitantes.

Es de gran importancia el estudio del LCR: tinciones, cultivos, estudios de biología molecular (reacción de polimerasa en cadena-RPC), tal como se señalaba en el artículo correspondiente (Capítulo III); sumado a los hallazgos de las neuroimágenes, particularmente la resonancia magnética (RM), por su mayor rendimiento en detectar lesiones. Si no se logra aclarar la etiología con métodos no invasores, se recomienda realizar biopsia cerebral.

Frente al diagnóstico de infección del SNC, una vez tomada la muestra de LCR, se recomienda el inicio de terapia empírica, que incluya al menos terapia antibacteriana (Tabla 1), antiviral (Tabla 2) y antifúngica (Tabla 3). Una vez orientado el diagnóstico etiológico por los hallazgos clínicos, microbiológicos, moleculares y de 
imágenes, se enfatiza la importancia de ajustar la terapia antiinfecciosa ${ }^{29,30}$ (Tabla 4).

\section{Neumonía}

Frente a la sospecha de una infección pulmonar en un $\mathrm{PHO}$, el médico clínico debe basar su diagnóstico diferencial en seis pilares fundamentales: historia, examen físico, exámenes generales, radiografía de tórax, tomografía (TAC) de tórax y evaluaciones etiológicas específicas, a través de técnicas no invasoras e invasoras con las herramientas de diagnóstico discutidas en profundidad en el Capítulo III.

Es fundamental establecer la relación del inicio de los síntomas sugerentes de infección pulmonar con el tipo, tiempo de aparición e intensidad del compromiso inmunológico $^{31}$. Se recomienda indagar, además, en los aspectos epidemiológicos: contactos, viajes, virus respiratorios circulantes y vacunas recibidas.

Los síntomas más frecuentes que orientan a una infección pulmonar en un $\mathrm{PHO}$ son fiebre, tos y polipnea ${ }^{32}$. El examen pulmonar podrá mostrar los clásicos signos de una neumopatía aguda, pero se debe tener en cuenta que el paciente neutropénico tiene una capacidad de respuesta inflamatoria muy escasa, por lo que la expresión clínica de su proceso infeccioso suele ser mínima.

En el examen extrapulmonar puede haber algunos hallazgos orientadores a agentes etiológicos específicos, tales como úlceras bucales y faríngeas que hacen sospechar virus de la familia Herpesviridae o lesiones necróticas de piel y mucosas en que $S$. aureus, $P$. aeruginosa y hongos filamentosos forman parte del diagnóstico diferencial ${ }^{33-35}$.

El enfoque terapéutico se hará de acuerdo al tipo de infiltrado pulmonar y su tiempo de aparición:

\section{Infiltrado focal}

Más frecuente en infecciones bacterianas: Streptococcus pneumoniae, Staphylococcus aureus, Klebsiella pneumoniae (Tabla 1). Si el paciente no presenta indicios de mejoría luego de $72 \mathrm{~h}$ de tratamiento, a pesar de una terapia antimicrobiana orientada a estos agentes, se sugiere considerar hongos, Nocardia sp, Actinomyces sp y micobacterias ${ }^{36}$ (Tabla 3).

\section{Infiltrado difuso}

Los infiltrados difusos se asocian, generalmente, a neumonías rápidamente progresivas, de alta mortalidad, por lo que requieren un enérgico enfrentamiento diagnóstico y terapéutico. Son causados por infecciones fúngicas invasoras como Aspergillus spp., agentes de mucormicosis o $P$. jirovecii o infecciones virales como CMV. En el diagnóstico diferencial de un infiltrado pulmonar difuso debe también considerarse Legionella sp, Mycoplasma sp y micobacterias ${ }^{37}$.

\section{Lesión nodular o cavitaria}

El diagnóstico diferencial incluye abscesos por $S$. aureus, $P$. aeruginosa, bacterias anaerobias, $M$. tuberculosis, infecciones fúngicas y la sobreinfección de un infarto pulmonar previo ${ }^{38}$.

\section{Efusión pleural}

La presencia de líquido en la cavidad pleural puede deberse a un fenómeno no infeccioso o ser secundario a una infección bacteriana: S. pneumoniae, S. aureus, $M$. tuberculosis ${ }^{1}$. Con mucha menor frecuencia, se ve efusión pleural en pacientes con infección por virus u hongos, incluido $P$. jirovecii.

\section{Estudio y decisiones terapéuticas}

El manejo ideal ante la sospecha clínica, es la realización de imágenes privilegiando la TAC de tórax sobre una radiología convencional, estudio etiológico considerando los exámenes microbiológicos presentados en el Capítulo II, incluyendo realización de lavado broncoalveolar (LBA) e inicio inmediato de terapia antimicrobiana empírica, acorde a las etiologías más probables dependiendo del tipo y grado de compromiso inmunológico y los patógenos prevalentes en esa situación clínica.

Es importante recalcar que el inicio de la terapia antimicrobiana en un $\mathrm{PHO}$ con sospecha de infección pulmonar es una emergencia; por lo tanto, la secuencia en el paciente no-neutropénico entre sospecha clínica, TAC, LBA e inicio de tratamiento no debe tomar más de $24 \mathrm{~h}$. Si existe imposibilidad de realizar un LBA en el momento en que el médico tratante lo solicitara, se deben tomar todas las muestras asequibles (hemocultivos, muestras de otros sitios, dependiendo de la orientación clínica), iniciar el manejo antimicrobiano empírico y esperar la respuesta clínica a las 48-72 h para decidir la realización o no de LBA, conducta que se adopta más por carencias de los sistemas de salud, sin ser la recomendación de este panel de expertos. La práctica de realizar siempre LBA antes de iniciar la antibioterapia, ofrece beneficios significativos. Es la única alternativa real de efectuar un tratamiento específico inicial, evitando una terapia empírica de muy amplio espectro, que en ocasiones incluye terapia antibacteriana, antifúngica, antiviral y antiparasitaria, con los consiguientes efectos adversos y gasto innecesario de recursos.

La realización de biopsia pulmonar en PHO (en reemplazo de LBA) se justifica en pacientes con infiltrado nodular. Requiere de equipamiento adecuado y cirujano experto en su realización.

\section{Terapia antibacteriana}

Debe incluir fármacos con acción antineumocócica, antiestafilocócica, antibacilos gramnegativos entéricos $\mathrm{y}$, dependiendo de la circunstancia epidemiológica y la condición de neutropenia, terapia anti $P$. aeruginosa. Si 
el paciente presenta un infiltrado pulmonar difuso debe, además, incluir terapia dirigida contra atípicos (Mycoplasma pneumoniae, Legionella sp, Chlamydia sp) (Tabla 1).

El tratamiento anti TBC o contra otras micobacterias no suele formar parte del manejo empírico inicial ${ }^{1,3,39}$.

\section{Terapia antifúngica}

Si se sospecha infección por Aspergillus sp, la indicación es iniciar voriconazol IV, terapia recomendada universalmente, con resultados superiores a anfotericina B deoxicolato, permitiendo disminuir mortalidad de $90 \%$ a 50\% (Tabla 3). Frente a un infiltrado pulmonar difuso iniciar, además, terapia anti $P$. jirovec $i i^{40,41}$

\section{Terapia antiviral}

Si se diagnostica una infección por virus herpes simplex, el tratamiento de elección es aciclovir IV. Si se diagnostica una infección por CMV, se recomienda ganciclovir como primera elección y foscarnet como alternativa en paciente con TPH neutropénico previo al implante ${ }^{1,3}$ (Tabla 2).

\section{Otros aspectos terapéuticos}

Si el paciente tiene una aspergilosis pulmonar invasora, se recomienda, además, el uso de factores estimulantes de colonias de granulocitos con el fin de acortar el período de neutropenia y considerar la cirugía precoz con extirpación de las lesiones pulmonares ante falta de respuesta, cercanía a grandes vasos o con la intención de rebajar la carga fúngica ante el requerimiento de terapias sucesivas que lleven a neutropenia profunda y prolongada ${ }^{42,43}$.

\section{Infecciones de piel y tejidos blandos}

Son infecciones frecuentes en $\mathrm{PHO}$, producidas por bacterias, virus y hongos, con manifestaciones clínicas generalmente atípicas o más graves que las observadas en pacientes inmunocompetentes, por la incapacidad de localizar la infección. El factor de riesgo más importante es la neutropenia profunda y prolongada, que se puede presentar, tanto en pacientes en quimioterapia como en receptores de $\mathrm{TPH}^{44}$.

Es relevante en estos pacientes establecer un buen diagnóstico, con una adecuada exploración clínica y adecuada obtención de muestra a través de una biopsia para estudio histológico, microbiológico (diagnóstico y susceptibilidad antimicrobiana cuando corresponda) y biología molecular a través de RPC.

Dependiendo del tipo de lesión y el tiempo de presentación, se sugiere asociar con determinadas etiologías más probables y recomendar, en consecuencia, distintas terapias empíricas en espera de los resultados de los estudios histológico, microbiológico y de biología molecular $(\mathrm{RPC} \mathrm{u} \text { otros })^{45}$.
Las infecciones en períodos iniciales de neutropenia ( $<7$ días) se presentan como erupción o celulitis en pliegues y áreas de disrupción cutánea y son, generalmente, causadas por bacterias grampositivas pertenecientes a la microbiota de la piel. Las etiologías más frecuentes son: S. aureus, Staphylococcus coagulasa negativa, Streptococcus pyogenes, Streptococcus del grupo viridans, Enterococcus, Corynebacterium spp, Clostridium spp y Bacillus spp.

Otra forma de presentación clínica en esta etapa son las lesiones vesiculosas, simples o hemorrágicas, que orientan a una infección por virus de la familia Herpesviridae, principalmente herpes simplex, VVZ y CMV, el que ocasionalmente adopta, además, otras formas de presentación, como nódulos y úlceras ${ }^{46,47}$.

Las infecciones tardías ( $\geq 7$ días de neutropenia) suelen presentarse como nódulos subcutáneos, lesiones necróticas, fascitis necrosante o ectima gangrenoso. Las etiologías más probables son bacilos gramnegativos o cocáceas grampositivas del ambiente intrahospitalario (con mayor probabilidad de resistencia a antimicrobianos), hongos levaduriformes (Candida spp) o filamentosos (Aspergillus, agentes de mucormicosis, Fusarium), Mycobacterium spp. y Nocardia $\mathrm{spp}^{34,48}$. Estas manifestaciones constituyen siempre formas graves de presentación clínica y es relevante su estudio etiológico e inicio de terapia antimicrobiana antes de $24 \mathrm{~h}$ de evolución.

Específicamente, en la etiología de la fascitis necrosante, es necesario considerar cocáceas grampositivas y bacilos gramnegativos, aeróbicos y anaeróbicos estrictos. Las bacterias más frecuentemente aisladas son Streptococcus spp, S. aureus, Pseudomonas spp, Stenotrophomonas maltophilia, Aeromonas spp, Escherichia coli, Enterococcus spp y anaerobios estrictos como Clostridium spp. El ectima gangrenoso, clásicamente es producido por Pseudomonas spp, describiéndose también casos de infección por $S$. aureus, Aeromonas spp, Serratia spp, $S$. maltophilia, Candida spp y otros hongos que incluyen Aspergillus spp, agentes de mucormicosis y Fusarium $\operatorname{spp}^{44,45}$.

El tratamiento antimicrobiano empírico debe basarse en la epidemiología de las infecciones en cada institución, la presentación clínica y la magnitud y duración de la neutropenia. Se sugiere cobertura antiestafilocócica (usualmente cloxacilina, clindamicina o vancomicina si hay antecedentes de Staphylococcus resistente a meticilina (cloxacilina) o epidemiología institucional con resistencia $>$ a $30 \%$ o alergia a $\beta$-lactámicos) más un agente específico con actividad frente a Pseudomonas spp, de acuerdo a la susceptibilidad in vitro (aminoglucósido, ceftazidima o cefepime, piperacilina/tazobactam o carbapenémico) (Tabla 1). Si la lesión sugiere una infección viral (vesículas, úlceras) incluir aciclovir si la posibilidad es herpes simplex o VVZ o ganciclovir si 
se piensa en CMV (Tabla 2). Si la lesión orienta a una infección fúngica (nódulos, lesión necrótica) se sugiere tratamiento con anfotericina B liposomal, voriconazol o equinocandinas (esta última si la sospecha es Candida spp.) (Tabla 3); si la etiología probable es Nocardia, se sugiere uso de cotrimoxazol IV ${ }^{1,3}$

\section{Tiflitis/enterocolitis neutropénica/enteritis neutropénica}

Cuadro de inflamación intestinal relacionado a injuria de la mucosa intestinal, provocada por fármacos citotóxicos, en el contexto de una neutropenia profunda y de deterioro de la respuesta del hospedero frente a microorganismos intestinales. Lo anterior provocaría invasión microbiana de la pared intestinal, con riesgo de necrosis y perforación.

Es la complicación intestinal más común en pacientes neutropénicos y el compromiso se produce preferentemente en el ciego, segmento de íleon terminal y colon ascendente ${ }^{49}$.

La incidencia, según revisiones sistemáticas, es de 5\% en adultos y 3 a $9 \%$ en niños, con una mortalidad variable en las diferentes series, que oscila entre 1 y $26 \%$.

La presentación clínica es de dolor y distensión abdominal, unido a vómitos y diarrea acuosa o sanguinolenta, en el contexto de un cuadro de neutropenia y fiebre. El diagnóstico se hace a través de sospecha clínica e imágenes: radiografía de abdomen, ecografía y TAC que muestren engrosamiento de la pared intestinal $>4 \mathrm{~mm}$.

La etiología es polimicrobiana acorde a la microbiota intestinal. Las bacterias gramnegativas más frecuentes son: E. coli, Klebsiella spp, Pseudomonas spp, Bacillus spp, y Bacteroides spp, sumado a algunas cocáceas grampositivas, como Enterococus spp, Streptococcus spp y otros anaerobios estrictos del tubo digestivo bajo.

Es necesario, además, considerar la posibilidad de bacteriemia por estos agentes, secundaria a traslocación bacteriana desde el tubo digestivo a la sangre y la presencia de candidemia, por igual mecanismo ${ }^{50,51}$.

En cuanto a la terapia global, existe consenso que, frente a la sospecha diagnóstica, se debe instaurar el reposo intestinal, alimentación parenteral y tratamiento antimicrobiano empírico de amplio espectro.

El esquema antimicrobiano a utilizar debe basarse en la epidemiología institucional, con fármacos activos frente bacilos gramnegativos entéricos, Pseudomonas spp $\mathrm{y}$ anaerobios estrictos del tubo digestivo bajo, incluyendo C. difficile. Se sugieren esquemas con $\beta$-lactámicos con acción anti Pseudomonas más aminoglucósidos más anti anaeróbicos: ceftazidima o cefepime + amikacina + metronidazol o monoterapia con piperacilina/tazobactam o un carbapenémico. Frente a la sospecha de cocáceas grampositivas resistentes, se sugiere considerar precozmente el uso de vancomicina o linezolid. Ante la sospecha o presencia de enterobacterias productoras de BLEE, se recomienda indicar carbapenémicos y frente a la presencia de enterobacterias productoras de carbapenemasas, considerar uso de colistín ${ }^{52}$ (Tabla 1).

El tratamiento antifúngico con un medicamento fungicida con actividad frente a Candida spp-equinocandinas deben utilizarse como primera línea- se recomienda prescribirlo si los hemocultivos son positivos para un hongo o si hay falta de respuesta terapéutica luego de $72 \mathrm{~h}$ de antibioterapia adecuada, con persistencia de la neutropenia, fiebre y manifestaciones clínicas sugerentes de persistencia de enterocolitis, o antes si la evolución es desfavorable $\mathrm{e}^{1,3}$ (Tabla 3). El uso de anti parasitarios es excepcional, acorde a la epidemiología local y a la presencia de síntomas específicos sugerentes de alguna parasitosis.

\section{Infecciones urinarias}

Son producidas por los mismos agentes que en el paciente inmunocompetente. Los más frecuentes son enterobacterias y Enterococcus spp. Se sugiere tratar de acuerdo a la epidemiología institucional y sus patrones de susceptibilidad antimicrobiana ${ }^{52}$ (Tabla 1).

Candida spp es también un posible agente etiológico en estos pacientes que tienen múltiples factores de riesgo; en caso de confirmarse una infección del tracto urinario por Candida spp, el tratamiento de elección es fluconazol, considerando su excelente concentración renal y en vías urinarias $^{1,3}$ (Tabla 3).

\section{Protocolo de obtención de muestra de para medir concentraciones plasmáticas de diferentes medicamentos en PHO}

Se recomienda la monitorización de concentraciones plasmáticas de vancomicina, amikacina y voriconazol ${ }^{54}$.

\section{Monitorización terapéutica de vancomicina}

\section{- Farmacocinética}

Tiempo de vida media (T1/2): 5-6 h (es menor en pediatría).

Excreción: 80-90\% inalterada por vía renal.

\section{- Farmacodinamia}

La acción bactericida de vancomicina ha demostrado tener un mayor efecto terapéutico cuando su área bajo la curva $(\mathrm{ABC})$ /concentración inhibitoria mínima (CIM) es mayor a $400 \mathrm{mg} / \mathrm{h} / \mathrm{L}$, y sus concentraciones basales plasmáticas se encuentran en 10-20 $\mu \mathrm{g} / \mathrm{ml}$. 


\section{Medición de concentraciones plasmáticas de vancomicina}

Se deben controlar las concentraciones plasmáticas de vancomicina luego de cinco vidas medias que, usualmente, corresponde a la dosis $\mathrm{N}^{\circ}$ 5-7 de un tratamiento de cada $6 \mathrm{~h}$. En forma convencional, se sugiere medir concentraciones plasmáticas luego de $48 \mathrm{~h}$ de iniciado el tratamiento.

La toma de muestra de concentraciones plasmáticas de vancomicina incluye:

- Concentración pico: la muestra se toma $30 \mathrm{~min}$ después de terminada la infusión normal, que habitualmente dura $1 \mathrm{~h}$.

- Concentración valle: la muestra se toma 30 min antes de la próxima dosis.

La monitorización incluye medición de creatininemia y nitrógeno ureico dos veces a la semana.

\section{Concentraciones plasmáticas óptimas de vancomicina}

Concentración pico: $25-40 \mu \mathrm{g} / \mathrm{ml}$.

Concentración valle: $10-15 \mu \mathrm{g} / \mathrm{ml}$. En condiciones especiales (sepsis grave, infección de SNC o CIM de bacterias elevado) consultado con equipo de Infectología y Farmacia Clínica, la concentración valle puede ser $15-20 \mu \mathrm{g} / \mathrm{ml}$.

Ambas concentraciones están sobre un ABC/CIM $>400 \mathrm{mg} / \mathrm{h} / \mathrm{L}$. Si dichas concentraciones no se alcanzan de forma óptima, se debe comunicar con equipo de Infectología y/o Farmacia Clínica ${ }^{55,56}$.

\section{Monitorización terapéutica de amikacina}

- Farmacocinética

T1/2: 2-3 h (es menor en pediatría)

Excreción: $80-90 \%$ inalterada por la vía renal.

Volumen de distribución: Bajo, aumenta en relación a infección grave.

- Farmacodinamia

La acción bactericida de amikacina ha demostrado tener un mayor efecto terapéutico cuando la concentración pico (Cmax) se encuentra 10 a 15 veces sobre la CIM $(\mathrm{Cmax} / \mathrm{CIM}>15)$ y sus valores pico se encuentran en $40-50 \mu \mathrm{g} / \mathrm{ml}$ en dosis única al día. Las concentraciones plasmáticas valle aseguran la monitorización de toxicidad del tratamiento acumulado.

\section{Medición de concentraciones plasmáticas de amikacina}

Se deben controlar concentraciones plasmáticas de amikacina luego de cinco vidas medias, lo que usualmente es en la $2^{\mathrm{a}}$ dosis en regímenes de administración cada $24 \mathrm{~h}$ y en la $3^{\mathrm{a}}$ dosis en regímenes cada $12 \mathrm{~h}$.
La obtención de muestra para medir concentraciones plasmáticas de amikacina incluye:

- Concentración pico tomar $30 \mathrm{~min}$ posterior a finalizar una infusión normal de $30 \mathrm{~min}$.

- Concentración valle: tomar 30 min antes de la próxima dosis.

La monitorización incluye la medición de creatininemia y nitrógeno ureico dos veces a la semana.

\section{Concentraciones plasmáticas óptimas \\ de amikacina}

Concentración pico: $30-50 \mu \mathrm{g} / \mathrm{ml}$.

Concentración valle: $<2 \mu \mathrm{g} / \mathrm{ml}$ en dosificación cada $24 \mathrm{~h}$.

Ambas concentraciones se controlan al menos dos veces por semana. Si las concentraciones plasmáticas no se alcanzan de forma óptima se debe comunicar a equipo de Infectología y/o Farmacia Clínica ${ }^{57}$.

\section{Monitorización terapéutica de voriconazol}

La toma de concentraciones plasmáticas terapéuticas incluye:

- Concentración pico: luego de 3-5 días de tratamiento, tomado $1 \mathrm{~h}$ después de finalizada la infusión de voriconazol y en el caso de la vía oral, 1 h post-administración. La concentración pico se encuentra relacionada con la toxicidad del tratamiento, especialmente con la fototoxicidad y hepatotoxicidad.

- Concentración plasmática tóxica de voriconazol: concentración peak $>9,0 \mu \mathrm{g} / \mathrm{ml}$.

- Concentración valle: luego de 3-5 días de tratamiento, la muestra para medición valle se obtiene $30 \mathrm{~min}$ antes de la próxima dosis. Esta concentración está relacionada con la eficacia del tratamiento.

- Concentración efectiva para profilaxis: $>0,5 \mu \mathrm{g} / \mathrm{ml}$.

- Rango terapéutico: 1- 5,5 $\mu \mathrm{g} / \mathrm{ml}$. Para tratamiento de aspergilosis invasora se sugiere concentraciones plasmáticas $\geq 2,0 \mu \mathrm{g} / \mathrm{ml}$.

Ajuste de dosis

- Tratamiento con voriconazol: En pediatría es frecuente observar dificultades en lograr concentraciones plasmáticas adecuadas. Si las concentraciones valle se encuentran $<1 \mu \mathrm{g} / \mathrm{ml}$ se recomienda ajustar dosis a $18 \mathrm{mg} / \mathrm{kg} /$ día, fraccionada cada ocho horas y controlar concentración plasmática cinco días después.

- Profilaxis con voriconazol: Si la concentración valle es $<0,5 \mu \mathrm{g} / \mathrm{ml}$, se recomienda ajustar la dosis a 10 $\mathrm{mg} / \mathrm{kg} /$ día, fraccionado cada $12 \mathrm{~h}$, vía oral, tomando en consideración que la presencia de enfermedad inflamatoria intestinal (ej.: Enfermedad injerto contra hospedero, enfermedad de Crohn) implica ajuste a tratamiento $\mathrm{IV}^{58-60}$. 
En conclusión, el enfrentamiento terapéutico de los $\mathrm{PHO}$ requiere una mirada integral, desde el conocimiento detallado de la situación del paciente, el riesgo que significa el episodio, la epidemiología institucional y la definición de procesos diagnósticos terapéuticos según la condición clínica del paciente, en los que se debe asegurar el adecuado estudio para precisar la etiología y el inicio precoz de terapias de mayor espectro a altas dosis, que deberá ajustarse según resultados del estudio microbiológico y la evolución del paciente. En nuestra opinión, es muy relevante para la práctica clínica en los hospitales públicos que atienden $\mathrm{PHO}$, contar con los protocolos terapéuticos aquí presentados, adaptados a su epidemiología, y que se aseguren en los arsenales de sus farmacias los antimicrobianos mencionados, estableciendo para las situaciones excepcionales de ausencia de alguno de estos fármacos, mecanismos expeditos de acceso a fin de cumplir con la premisa máxima de terapia oportuna.

\section{Resumen}

Este documento incluye los recursos terapéuticos antiinfecciosos necesarios para pacientes inmunocomprometidos por terapia de cáncer o receptores de trasplante de precursores hematopoyéticos (TPH). Se aborda la terapia indicada para pacientes con las situaciones clínicas prevalentes en esta población y la terapia indicada para algunos microorganismos específicos. Según presentación clínica, se aborda el manejo de pacientes con: neutropenia febril sin foco, sepsis, infecciones del sistema nervioso central, neumonía, infecciones de piel y tejidos blandos, enterocolitis neutropénica e infección del tracto urinario. Se describe el arsenal terapéutico necesario, las dosis recomendadas y las precauciones especiales para el uso de antibacterianos, antivirales, antifúngicos y antiparasitarios en esta población, incluida la medición de concentraciones plasmáticas de ciertos fármacos en situaciones específicas.

Tabla 1. Dosis, intervalo y vía de administración de antibacterianos en niños y adultos con neutropenia febril

\begin{tabular}{|c|c|c|c|c|c|}
\hline Antimicrobiano & $\begin{array}{l}\text { Dosis niños* } \\
\text { (mg/kg/día) }\end{array}$ & $\begin{array}{l}\text { Dosis adultos } \\
\text { (día) }\end{array}$ & $\begin{array}{c}\text { Vía de } \\
\text { administración }\end{array}$ & $\begin{array}{c}\text { Frecuencia de } \\
\text { administración }(\mathrm{h})\end{array}$ & Comentarios \\
\hline \multicolumn{6}{|l|}{$\beta$ lactámicos } \\
\hline Amoxicilina/ácido clavulánico & $50-100$ & $1.750 / 250 \mathrm{mg}$ & VO & $8-12$ & Dosis en base a amoxicilina \\
\hline Ampicilina & $100-400$ & $8-12 \mathrm{~g}$ & IV & $4-6$ & Dosis altas en infección del SNC \\
\hline Cefazolina & 100 & $4-6$ & IV & $6-8$ & Alternativa a cloxacilina \\
\hline Cefepima & $100-150$ & $6 \mathrm{~g}$ & IV & 8 & Dosis altas en infección del SNC \\
\hline Cefixima & 8 & $400 \mathrm{mg}$ & VO & 24 & \\
\hline Cefotaxima & $100-300$ & $6-12 \mathrm{~g}$ & IV & $4-6-8$ & Dosis altas en infección del SNC \\
\hline Ceftazidima & $100-150$ & $6 \mathrm{~g}$ & IV & 8 & Dosis altas en infección del SNC \\
\hline Ceftriaxona & $50-100$ & $2-4 \mathrm{~g}$ & IV & $12-24$ & Dosis altas en infección del SNC \\
\hline Cefuroxima-acetil & $30-50$ & $1 \mathrm{~g}$ & Vo & 12 & \\
\hline Cloxacilina & 100 & $2 \mathrm{~g}(\mathrm{VO}) 12 \mathrm{~g}(\mathrm{IV})$ & IVNO & $4-6$ & \\
\hline Imipenem & $60-100$ & $2 \mathrm{~g}$ & IV & 6 & Dosis altas en infección del SNC \\
\hline Meropenem & $60-120$ & $3-6 \mathrm{~g}$ & IV & 8 & Dosis altas en infección del SNC \\
\hline Penicilina G & $100.000-250.000 \mathrm{U}$ & 24 millones $U$ & IV & $4-6$ & \\
\hline Piperacilina/tazobactam & $100-400$ & $13,5 \mathrm{~g}$ & IV & $8-6$ & Dosis altas en infección del SNC \\
\hline \multicolumn{6}{|l|}{ Aminoglucósidos } \\
\hline Amikacina & $15-30$ & $15 \mathrm{mg} / \mathrm{kg}$ & IV & 24 & $\begin{array}{l}\text { Monitorear concentraciones plasmáticas } \\
\text { Dosis altas en infección del SNC }\end{array}$ \\
\hline Gentamicina & $3-5$ & $1,7 \mathrm{mg} / \mathrm{kg}$ & IV & 8 & $\begin{array}{l}\text { Monitorear concentraciones plasmáticas } \\
\text { Dosis altas en infección del SNC }\end{array}$ \\
\hline \multicolumn{6}{|l|}{ Glucopéptidos } \\
\hline Vancomicina & $40-60$ & $2-4 \mathrm{~g}$ & IV & $6-8-12$ & $\begin{array}{l}\text { Monitorear concentraciones plasmáticas } \\
\text { Dosis altas en infección del SNC }\end{array}$ \\
\hline
\end{tabular}




\begin{tabular}{|c|c|c|c|c|c|}
\hline \multicolumn{6}{|c|}{ Macrólidos azálidos } \\
\hline Azitromicina & 10 & $500 \mathrm{mg}$ & VO & 24 & \\
\hline Claritromicina & $10-20$ & $1 \mathrm{~g}$ & VO & 12 & \\
\hline Eritromicina & $40-50$ & $250-500 \mathrm{mg}$ & VO & 6 & \\
\hline \multicolumn{6}{|l|}{ Quinolonas } \\
\hline Ciprofloxacina & $20-40$ & $\begin{array}{c}1.000 \mathrm{mg}(\mathrm{VO}) \\
800 \mathrm{mg}(\mathrm{IV})\end{array}$ & IVNO & $8-12$ & Oral: separado de lácteos $>1 \mathrm{~h}$ \\
\hline Levofloxacina & $8-10$ & $750 \mathrm{mg}$ & IVNO & $12-24$ & Oral: separado de lácteos $>1 \mathrm{~h}$ \\
\hline Moxifloxacina & $10-15$ & $400 \mathrm{mg}$ & VO & 24 & Oral: separado de lácteos $>1 \mathrm{~h}$ \\
\hline \multicolumn{6}{|l|}{ Sulfas } \\
\hline Cotrimoxazol & $\begin{array}{c}100 \mathrm{mg} \text { de sulfa/ } \\
20 \mathrm{mg} \text { de trimetoprim }\end{array}$ & $\begin{array}{l}15-20 \mathrm{mg} / \mathrm{kg} / \mathrm{dí} \\
\text { trimetroprim }\end{array}$ & IVNO & 6 & Dosis para enfermedad por $P$. jirovecii \\
\hline \multicolumn{6}{|l|}{ Otros } \\
\hline Clindamicina & $20-40$ & $2.700 \mathrm{mg}$ & IV & $6-8$ & \\
\hline Colistin** & $2,5-5 \mathrm{~m} \mathrm{CBA}$ & $\begin{array}{l}9 \text { millones UI equivalente } \\
\text { a } 300 \mathrm{mg} \text { de CBA }\end{array}$ & IV & 8 & Se recomienda \\
\hline Daptomicina & $\begin{array}{l}\text { 2-5 años: } 10 \\
\text { 6-11 años: } 7\end{array}$ & $4-6 \mathrm{mg} / \mathrm{kg}$ & IV & 24 & \\
\hline Etambutol & $10-25$ & $15-25 \mathrm{mg} / \mathrm{kg}$ & VO & 24 & \\
\hline Isoniazida & $10-15$ & $10-15 \mathrm{mg} / \mathrm{kg}$ & VO & 24 & \\
\hline Linezolid & 30 & $1.200 \mathrm{mg}$ & IVNO & $8-12$ & \\
\hline Metronidazol & $30-50$ & $1.500 \mathrm{mg}$ & IVNO & 8 & \\
\hline Pirazinamida & $15-30$ & $25 \mathrm{mg} / \mathrm{kg}$ & VO & 24 & \\
\hline Rifampicina & $10-20$ & $600 \mathrm{mg}$ & VO/IV & $12-24$ & \\
\hline Tigeciclina & $50 \mathrm{mg} / \mathrm{d}$ & $100 \mathrm{mg}$ & IV & 12 & \\
\hline
\end{tabular}

\section{Tabla 2. Dosis, intervalo y vía de administración de antivirales en niños y adultos con neutropenia febril}

\begin{tabular}{|c|c|c|c|c|c|}
\hline Antiviral & $\begin{array}{l}\text { Dosis niños } \\
\text { (mg/kg/día) }\end{array}$ & $\begin{array}{l}\text { Dosis adultos } \\
\text { (día) }\end{array}$ & $\begin{array}{c}\text { Vía de } \\
\text { administración }\end{array}$ & $\begin{array}{c}\text { Frecuencia de } \\
\text { administración (h) }\end{array}$ & Comentarios \\
\hline Aciclovir & $\begin{array}{l}30-60 \\
60-80\end{array}$ & $30 \mathrm{mg} / \mathrm{kg}$ & $\begin{array}{l}\text { IV } \\
\text { VO }\end{array}$ & $\begin{array}{c}8 \\
6-8\end{array}$ & \\
\hline Valaciclovir & $>3 \mathrm{~m}: 40 / 60$ & $2.000 \mathrm{mg}$ & VO & $8-12$ & $\begin{array}{l}40 \mathrm{mg}: \text { VHS } \\
60 \mathrm{mg}: \text { VVZ }\end{array}$ \\
\hline Ganciclovir & $10-15$ & $10 \mathrm{mg} / \mathrm{kg}$ & IV/IV & $12-24$ & 5 mg/kg/día en mantención \\
\hline Valganciclovir & $20-50$ & $1.800 \mathrm{mg}$ & VO & $12-24$ & \\
\hline Cidofovir & $0,5-5$ & $5 \mathrm{mg} / \mathrm{kg}$ semanal & IV & 1 vez/semana & Igual dosis total fraccionada 3 veces/semana \\
\hline Foscarnet & $120-180 \mathrm{mg}$ & $180 \mathrm{mg}$ & IV & $8-12$ & $\begin{array}{l}120 \text { mg: VHS } \\
180 \text { mg: CMV/NVZ }\end{array}$ \\
\hline Oseltamivir & $\begin{array}{c}\text { < } 1 \text { a: } 6 \text { mg/kg/día } \\
>1 \text { año = dosis/día } \\
>1 \text { a < } 15 \text { kg: } 30 \mathrm{mg} \\
\text { 16-23 kg: } 45 \mathrm{mg} \\
\text { 24-40 kg: } 60 \mathrm{mg} \\
>40 \mathrm{~kg}: 75 \mathrm{mg}\end{array}$ & $150 \mathrm{mg}$ & VO & 12 & \\
\hline
\end{tabular}


Tabla 3. Dosis, intervalo y vía de administración de antifúngicos en niños y adultos con neutropenia febril

\begin{tabular}{|c|c|c|c|c|c|}
\hline Antifúngico & $\begin{array}{l}\text { Dosis niños } \\
\text { (mg/kg/día) }\end{array}$ & $\begin{array}{l}\text { Dosis adultos } \\
\text { (día) }\end{array}$ & $\begin{array}{c}\text { Vía de } \\
\text { administración }\end{array}$ & $\begin{array}{c}\text { Frecuencia de } \\
\text { administración (h) }\end{array}$ & Comentarios \\
\hline \multicolumn{6}{|l|}{ Anfotericinas } \\
\hline Anfotericina complejo lipídico & $2,5-5$ & $3-5 \mathrm{mg} / \mathrm{kg}$ & IV & 24 & \\
\hline Anfotericina dispersión coloidal & $3-5$ & $3-5 \mathrm{mg} / \mathrm{kg}$ & IV & 24 & \\
\hline Anfotericina liposomal & $3-5$ & $3-5 \mathrm{mg} / \mathrm{kg}$ & IV & 24 & Dosis altas en infección del SNC \\
\hline \multicolumn{6}{|l|}{ Azoles } \\
\hline Fluconazol & 12 & $400-800 \mathrm{mg}$ & IV/oral & 24 & \\
\hline Itraconazol & $3-8$ & $200-400 \mathrm{mg}$ & Oral & $12 / / 24$ & Pobre biodisponibilidad \\
\hline Posaconazol & $\begin{array}{c}\text { } \leq 34 \mathrm{~kg}: 6-12 \\
>34 \mathrm{~kg}: 200 / 400\end{array}$ & $800 \mathrm{mg}$ & Oral & $\begin{array}{l}6 / 12 \\
6 / 12\end{array}$ & Monitorear concentraciones plasmáticas \\
\hline Voriconazol & $16-18$ & $\begin{array}{c}12 \mathrm{mg} / \mathrm{kg} \text { (carga) } \\
8 \mathrm{mg} / \mathrm{kg} \text { (mantención) }\end{array}$ & IV/oral & $8-12$ & Monitorear concentraciones plasmáticas \\
\hline \multicolumn{6}{|l|}{ Equinocadinas } \\
\hline Anidulafungina & $1,5 / 3$ & $200 \mathrm{mg} / 100 \mathrm{mg}$ & IV & 24 & Dosis de carga/mantención \\
\hline Caspofungina & $70 / 50 \mathrm{mg} / \mathrm{m}^{2}$ & $70 \mathrm{mg} / 50 \mathrm{mg}$ & IV & 24 & Dosis de carga/mantención \\
\hline Micafungina & $2-4$ & $100 \mathrm{mg}$ & IV & 24 & \\
\hline
\end{tabular}

Tabla 4. Dosis, intervalo y vía de administración de antiparasitarios en niños y adultos con neutropenia febril

\begin{tabular}{|c|c|c|c|c|c|}
\hline Antiparasitario & $\begin{array}{l}\text { Dosis niños } \\
\text { (mg/kg/día) }\end{array}$ & $\begin{array}{l}\text { Dosis adultos } \\
\text { (día) }\end{array}$ & $\begin{array}{c}\text { Vía de } \\
\text { administración }\end{array}$ & $\begin{array}{c}\text { Frecuencia de } \\
\text { administración (h) }\end{array}$ & Comentarios \\
\hline Benznidazol & 10 & $5-7 \mathrm{mg} / \mathrm{kg}$ & vo & 24 & \\
\hline Ivermectina & 3-9 mg* & $200 \mu \mathrm{g} / \mathrm{kg}$ & VO & 24 & Dosis total diaria \\
\hline Metronidazol & $30-50$ & 1.500 & IVNO & 8 & \\
\hline Nitazoxanida & $100-200 \mathrm{mg}^{*}$ & $1.000 \mathrm{mg}$ & VO & 12 & Dosis total diaria \\
\hline Nifurtimox & $12,5-20$ & $15 \mathrm{mg} / \mathrm{kg}$ & VO & $8-12$ & c/8 h en pediatría \\
\hline Pirimetamina & $1-2$ & $\begin{array}{c}200 \text { mg (carga) } \\
75 \text { mg (mantención) }\end{array}$ & vo & 24 & \\
\hline Sulfadiazina & 75-150 (de sulfa) & $\begin{array}{l}<60 \mathrm{~kg}: 4 \mathrm{~g} \\
\geq 60 \mathrm{~kg}: 6 \mathrm{~g}\end{array}$ & IV & 6 & \\
\hline Tinidazol & 50 & $2 \mathrm{~g}$ & VO & 24 & \\
\hline
\end{tabular}

\section{Referencias bibliográficas}

1.- Freifeld A G, Bow E J, Sepkowitz K A, Boeckh $\mathrm{M}$ J, Ito J I, Mullen C A, et al. Clinical practice guideline for the use of antimicrobial agents in neutropenic patients with cancer: 2010 update by the Infectious Diseases Society of America. Clin Infect Dis 2011; 52: e56-93. DOI: $10.1093 / \mathrm{cid} / \mathrm{ciq} 147$.

2.- Phillips R S, Lehrnbecher T, Alexander S,
Sung L. Updated systematic review and metaanalysis of the performance of risk prediction rules in children and young people with febrile neutropenia. PLoS One 2012; 7: $38300 \mathrm{https://}$ doi.org/10.1371/journal.pone.0038300.

3.- Lehrnbecher T, Robinson P, Fisher B T, Alexander S, Amman R, Beauchemin M, et al. Guidelines for the management of fever and neutropenia in children with cancer and hematopoietic stem cell transplantation. Update
2017. J Clin Oncol 2017; 35 (18): 2082-94 DOI: 10.1200/JCO.2016.71.7017.

4.- Klastersky J, Paesmans M, Rubenstein E B, Boyer M, Elting L, Feld R, et al. The Multinational Association for Supportive Care in Cancer Risk Index: a multinational scoring system for identifying low-risk febrile neutropenic cancer patients. J Clin Oncol 2000; 18: 3038-51. DOI: 10.1200/ JCO.2000.18.16.3038. 
5.- Palazzi D L. The use of antimicrobial agents in children with fever during chemotherapy induced neutropenia: the importance of risk stratification. Pediatr Infect Dis J 2011; 30: 887-90. DOI: 10.1097/INF.0b013e3182311343.

6.- Taplitz R A, Kennedy E B, Bow E J, Crews J, Gleason C, Hawley D K, et al. Outpatient management of fever and neutropenia in adults treated for malignancy: American Society of Clinical Oncology and Infectious Diseases Society of America Clinical Practice Guideline Update. J Clin Oncol 2018; 36: 1443 53. DOI: 10.1200/JCO.2017.77.6211.

7.- Klastersky J, Awada A, Paesmans M, Aoun M. Febrile neutropenia: a critical review of the initial management. Crit Rev Oncol Hematol 2011; 78: 185-94. DOI: 10.1016/j. critrevonc.2010.03.008.

8.- Santolaya M E, Álvarez A M, Becker A, Cofré J, Enríquez N, Paya E, et al. Prospective, multicenter evaluation of risk factors associated with invasive bacterial infection in children with cancer, neutropenia, and fever. J Clin Oncol 2001; 19: 3415-21. DOI: 10.1200/ JCO.2001.19.14.3415.

9.- Orme L M, Babl F E, Barnes C, Barnett P, Donath S, Ashley D M. Outpatient versus inpatient IV antibiotic management for pediatric oncology patients with low risk febrile neutropenia: a randomized trial. Pediatric Blood \& Cancer 2014; 61: 1427-33. DOI: 10.1002/ pbc. 25012.

10.- Santolaya M E, Álvarez A M, Acuña M, Avilés C L, Salgado C, Tordecilla J, et al. Efficacy and safety of withholding antimicrobial treatment in children with cancer, fever and neutropenia, with a demonstrated viral respiratory infection: a randomized clinical trial. Clin Microbiol Infect 2017; 23 (3): 173-8. DOI: 10.1016/j. cmi.2016.11.001.

11.- Paganini H, Santolaya M E, Álvarez M, Araña M, Arteaga R, Bonilla A, et al. Diagnóstico y tratamiento de la neutropenia febril en niños con cáncer. Consenso de la Sociedad Latinoamericana de Infectología Pediátrica. Rev Chil Infect 2011; 28 (Supl 1): 10-43. http://dx.doi.org/10.4067/S071610182011000400003.

12.- Rabagliati R, Bertín P, Cerón I, Rojas H, Domínguez I, Vera A, et al. 1. Epidemiology of febrile neutropenia in adult patients with acute leukemia and lymphoma; Cohort study of public and private hospital of Santiago, Chile. Rev Chilena Infectol 2014; 31: 721-8. DOI: $10.4067 / \mathrm{S} 0716-10182014000600013$.

13.- Styczynski J, Czyzewski K, Wysocki M, Gryniewicz-Kwiatkowska O, KolodziejczykGietka A, Salamonowicz M, et al. Increased risk of infections and infection-related mortality in children undergoing haematopoietic stem cell transplantation compared to conventional anticancer therapy: a multicentre nationwide study. Clin Microbiol Infect 2016; 22: 179-83. DOI: 10.1016/j.cmi.2015.10.017.

14.- Fisher P G. It is time for pediatric oncology to grow up. J Clin Oncol 2018; 36 (10): 933-34. DOI: $10.1200 / J C O .2017 .77 .4307$.

15.- Santolaya M E, Álvarez A M, Avilés C L, Becker A, Mosso C, O’Ryan M, et al. Admission clinical and laboratory factors associated with death in children with cancer during a febrile neutropenic episode. Pediatr Infect Dis J 2007; 26: 794-8. DOI: 10.1097/ INF.0b013e318124aa44.

16.- Ascioglu S, Rex J H, de Pauw B, Bennett J E, Bille J, Crokaert F, et al. Defining opportunistic invasive fungal infections in immunocompromised patients with cancer and hematopoietic stem cell transplants: an international consensus. Clin Infect Dis 2002; 34: 7-14. DOI: 10.1086/323335.

17.- De Pauw B, Walsh T, Donnelly J, Stevens D A, Edwards J E, Calandra T, et al. European Organization for Research and Treatment of Cancer/Invasive Fungal Infections Cooperative Group. Revised definitions of invasive fungal disease from the European Organization for Research and Treatment of Cancer/Invasive Fungal Infections Cooperative Group and the National Institute of Allergy and Infectious Diseases Mycoses Study Group (EORTC/MSG) Consensus Group. Clin Infect Dis 2008; 46: 1813-21. DOI: $10.1086 / 588660$.

18.- Santolaya M E, Álvarez A M, Avilés C L, Becker A, Venegas M, Salgado C, et al. Prospective validation of a risk prediction model for severe sepsis in children with cancer and high-risk febrile neutropenia. Pediatr Infect Dis J 2013; 32: 1318-23. DOI: 10.1097/01. inf.0000436128.49972.16.

19.- McCavit T L, Winick N. Time-to-antibiotic administration as a quality of care measure in children with febrile neutropenia: a survey of pediatric oncology centers. Pediatr Blood Cancer 2012; 58: 303-5. DOI: 10.1002/ pbc. 23148

20.- Fletcher M, Hodgkiss H, Zhang S, Browning R, Hadden C, Hoffman T, et al. Prompt administration of antibiotics is associated with improved outcomes in febrile neutropenia in children with cancer. Pediatr Blood Cancer. 2013; 60: 1299-306. DOI: 10.1002/pbc.24485.

21.- Sano H, Kobayashi R, Suzuki D, Kishimoto K, Yasuda K, Kobayashi K. Comparison between piperacillin/tazobactam and cefepime monotherapies as an empirical therapy for febrile neutropenia in children with hematological and malignant disorders: A prospective, randomized study. Pediatr Blood Cancer 2015; 62: 356-8. PMID: 25251104.

22.- Robinson P D, Lehrnbecher T, Phillips R, Dupuis L, Sung L. Strategies for empiric management of pediatric fever and neutropenia in patients with cancer and hematopoietic stem- cell transplantation recipients: A systematic review of randomized trials. J Clin Oncol 2016; 34: 2054-60. DOI: 10.1200/JCO.2015.65.8591.

23.- Falagas M E, Tansarli G S, Rafailidis $P$ I, Kapaskelis A, Vardakas K Z. Impact of antibiotic MIC on infection outcome in patients with susceptible Gram-negative bacteria: a systematic review and meta-analysis. Antimicrob Agents Chemother 2012; 56: 4214 22. DOI: $10.1128 /$ AAC.00663-12.

24.- Zunt J R. Central nervous system infection during immunosuppression. Neurol Clin 2002; 20 (1): 1-22. PMCID: PMC2695971.

25.- Martino R, Cordonier C. Toxoplasmosis following allogenic hematopoietic stem cell transplantation. Bone Marrow Transplant 2003; 31 (7): 617-8. DOI: 10.1038/sj.bmt.1703914.

26.- Brouwer M C, Tunkel A R, van de Beek D. Brain abscess. N Engl J Med 2014; 371: 44756. DOI: 10.1056/NEJMc1410501.

27.- Chiang S, Ahmad K, Milstein M. Treatment outcomes of chilhood tuberculosis meningitis. A systematic review and meta-analysis. Lancet Infect Dis 2014; 14: 947-57. DOI: 10.1016/ S1473-3099(14)70852-7.

28.- Schmidt M, Sonneville R, Schnell D, Bigé N, Hamidfar R, Mongardon N, et al. Clinical features and outcomes in patient with disseminated toxoplasmosis admited to intensive care; a multicenter study. Clin Infect Dis 2013: 57 : $1535-41$. DOI: $10.1093 / \mathrm{cid} /$ cit557.

29.- Reed C, Bryant R, Ibrahim A, Edwards J Jr, Filler S G, Goldberg R, et al. Combination polyene-caspofungin treatment of rhino orbital - cerebral mucormycosis. Clin Infect Dis 2008; 47: 364-71. DOI: 10.1086/589857.

30.- Ozsevik S N, Sensoy G, Karli A, Albayrak C, Dagdemir A, Belet N, et al. Invasive fungal infections in children with hematologic and malignant diseases. J Pediatr Hematol Oncol 2015; 37: 69-72. DOI: 10.1097/ MPH.0000000000000225.

31.- Batra S, Li B, Underhill N, Maloney R, Katz B Z, Hijiya N, et al. Clinical utility of bronchoalveolar lavage and respiratory tract biopsies in diagnosis and management of suspected invasive respiratory fungal infections in children. Pediatr Blood Cancer 2015; 62: 1579-86. https://doi.org/10.1002/pbc.25570.

32.- Torres J P, de la Maza V, Kors L, Villarroel M, Piemonte P, Izquierdo G, et al. Respiratory viral infections and coinfections in children with cancer, fever and neutropenia: Clinical outcome of infections caused by different respiratory viruses. Pediatr Infect Dis J 2016; 35: 949-54. DOI: $10.1097 /$ INF.0000000000001209.

33.- Barton C D, Waugh L K, Nielsen M J, Paulus S. Febrile neutropenia in children treated for malignancy. J Infect 2015; 71 (Suppl. 1): S27e35. DOI: 10.1016/j.jinf.2015.04.026. 
34.- Cohn S M, Pokala H R, Siegel J D, McClay J E, Leonard D, Kwon J, et al. Application of a standardized screening protocol for diagnosis of invasive mold infections in children with hematologic malignancies. Support Care Cancer 2016; 24: 5025-33 DOI: 10.1007/ s00520-016-3367-z.

35.- McCarthy M, Rosengart A, Schuetz A N, Kontoyiannis D P, Walsh T J. Mold infections of the central nervous system. N Engl J Med 2014; 371: 150-60. DOI: 10.1056/ NEJMra1216008.

36.- Torre Cisneros J, Doblas A, Aguado J M, San Juan R, Blanes M, Montejo M, et al. Tuberculosis after solid organ transplant: Incidence risk factors and clinical characteristics in the RESITRA cohort. Clin Infect Dis 2009; 48 (9): 1657-65. DOI: $10.1086 / 599035$.

37.- Fortún J, Carratalá J, Gavaldác J, Lizasoain M, Salavert M, de la Cámara R, et al. Recomendaciones sobre el tratamiento de la enfermedad fúngica invasiva por Aspergillus spp. y otros hongos filamentosos. Sociedad Española de Enfermedades Infecciosas y Microbiología Clínica (SEIMC). Actualización 2011. Enferm Infecc Microbio Clin 2011; 29: 435-54. doi:10.1016/j.eimc.2011.01.010.

38.- Perfect J, Hachem R, Wingard J R. Update on epidemiology and preventive strategies for invasive fungal infections in cancer patients. Clin Infect Dis 2014; 59 (S5): 352-5. DOI: $10.1093 / \mathrm{cid} / \mathrm{ciu} 639$

39.- Miedema K G, Tissing W J, Abbink F C, Ball L M, Michiels E M, van Vliet M J, et al. Riskadapted approach for fever and neutropenia in paediatric cancer patients - A national multicentre study. Eur J Cancer 2016; 53: 1624. DOI: 10.1016/j.ejca.2015.10.065.

40.- Katragkou A, Roilides E. Best practice in treating infants and children with proven, probable or suspected invasive fungal infections. Curr Opin Infect Dis 2011; 24: 225 9. DOI: $10.1097 / \mathrm{QCO} .0 \mathrm{~b} 013 \mathrm{e} 3283460 \mathrm{e} 22$.

41.- Neofytos D, Horn D, Annaisie E, Steinbach W, Olyaei A, Fishman J, et al. Epidemiology and outcome of invasive fungal infection in adult hematopoietic stem cell transplant recipients: analysis of Multicenter Prospective Multicenter Antifungal Therapy (PATH) Alliance Registry. Clin Infect Dis 2009; 48: 265-73. DOI: $10.1086 / 595846$.

42.- Walsh T J, Anaissie E J, Denning D W, Herbrecht R, Kontoyiannis D P, Marr K A, et al. Treatment of aspergillosis: Clinical practice guidelines of the Infectious Diseases Society of America. Clin Infect Dis 2008; 46: 327-60. DOI: $10.1086 / 525258$.

43.- Marr K, Schlamm H, Herbrecht R, Rottinghaus S T, Bow E J, Cornely OA, et al. Combination antifungal therapy for invasive fungal aspergillosis. Ann Intern Med 2015; 162: 81-92. DOI: $10.7326 / \mathrm{M} 13-2508$.

44.- Johnston D L, Waldhausen J H, Park J R. Deep soft tissue infections in the neutropenic pediatric oncology patient. J Pediatr Hematol Oncol 2001; 23: 443-7. PMID: 11878579.

45.- Stevens D L, Bisno Alan L, Chambers Henry F. Practice guidelines for the diagnosis and management of skin and soft tissue infections: 2014 update by the Infectious Diseases Society of America. Clin Infect Dis 2014:59(2): e1052. DOI: $10.1093 / \mathrm{cid} / \mathrm{ciu} 296$.

46.- Nesher L, Rolston K. The current spectrum of infection in cancer patients with chemotherapy related neutropenia. Infection 2014; 42: 5-13. DOI: $10.1007 / \mathrm{s} 15010-013-0525-9$.

47.- Solís Y, Álvarez A M, Fuentes D, de la Barra D, Avilés CL, Becker A, et al. Agentes causantes de bacteriemia en niños con cáncer y neutropenia febril de alto riesgo en seis hospitales de Santiago, Chile, período 2004- 2009. Rev Chil Infect 2012; 29 (2): 156-62. http://dx.doi.org/10.4067/S071610182012000200006.

48.- Dvorak C, Fisher B, Sung L. Antifungal prophylaxis in pediatric Hematology/ Oncology: new choices \& new data. Pediatr Blood Cancer 2012; 59: 21-6. DOI: 10.1002/ pbc. 23415 .

49.- Li K, Zheng S, Dong K, Gao Y, Wang H, Liu $\mathrm{G}$, et al. Diagnosis and outcome of neutropenic enterocolitis: experience in a single tertiary pediatric surgical center in China. Pediatr Surg Int 2011; 27: 1191-5. doi:10.1007/s00383-0112938-9

50.- Krones E, Högenauer C. Diarrhea in the immunocompromised patient. Gastroenterol Clin North Am. 2012; 41: 677-701. DOI: $10.1016 /$ j.gtc.2012.06.009.

51.- Gorschlüter M, Mey U, Strehl J, Schmitz $\mathrm{V}$, Rabe C, Pauls K, et al. Invasive fungal infections in neutropenic enterocolitis: A systematic analysis of pathogens, incidence, treatment and mortality in adult patients. BMC Infect Dis 2006, 6: 35-8. DOI: 10.1186/14712334-6-35.

52.- Sundell N, Boström H, Edenholm M, Abrahamsson J. Management of neutropenic enterocolitis in children with cancer.
Acta Paediatrica 2012; 101: 308-12. DOI: 10.1111/j.1651-2227.2011.02465.x.

53.- Sandoval C, Sinaki B, Weiss R, Munoz J, Ozkaynak M F, Tugal O, et al. Urinary tract infections in pediatric oncology patients with fever and neutropenia. Pediatr Hematol Oncol 2012; 29: 68-72. DOI: $10.3109 / 08880018.2011 .617809$.

54.- Lortholary O, Lefort A, Tod M, Chomat A M, Darras-Joly C, Cordonnier C, et al. Pharmacodynamics and pharmacokinetics of antibacterial drugs in the management of febrile neutropenia. Lancet Infect Dis 2008; 8: 612-20. DOI: 10.1016/S1473-3099(08)70228-7.

55.- Villena R, González C A, Nalegach M E, Vásquez A, Villareal M. Monitoreo terapéutico de vancomicina intravenosa en una unidad de paciente crítico pediátrico. Rev Chilena Infectol 2014; 31: 249-53. https://scielo.conicyt.cl/pdf/ rci/v31n3/art01.pdf.

56.- Acuña C, Morales J, Castillo C, Torres J P. Farmacocinética de vancomicina en niños hospitalizados en una unidad de paciente crítico. Rev Chilena Infectol 2013; 30: 585-90. DOI: $10.4067 / \mathrm{S} 0716-10182013000600001$.

57.- Downes K J, Hahn A, Wiles J, Courter J D, Vinks AA. Dose optimisation of antibiotics in children: application of pharmacokinetics/pharmacodynamics in paediatrics. Int J Antimicrob Agents. Elsevier B.V. 2014; 43: 223-30. DOI: 10.1016/j. ijantimicag.2013.11.006.

58.- Neely M, Rushing T, Kovacs A, Jelliffe R, Hoffman J. Voriconazole pharmacokinetics and pharmacodynamics in children. Clin Infect Dis 2010; 50: 27-36. DOI: 10.1086/648679

59.- Valenzuela R, Torres J P, Salas C, Gajardo I, Palma J, Catalán P, et al. Drug interaction of voriconazole-cyclosporine in children undergoing hematopoietic stem cell transplantation. Rev Chilena Infectol 2017; 34: 14-8. DOI: 10.4067/S071610182017000100002 .

60.- Pascual A, Calandra T, Bolay S, Buclin T, Bille J, Marchetti O. Voriconazole therapeutic drug monitoring in patients with invasive mycoses improves efficacy and safety outcomes. Clin Infect Dis 2008; 46: 201-11. DOI: $10.1086 / 524669$.

61.- Rosales R, Rojas L, Zamora F, Izquierdo G, Benavides C, González C. The challenge in colistin dosing: Updating available recommendations. Rev Chilena Infectol 2018; 35:105-16. DOI: 10.4067/s071610182018000200105 . 